\title{
Septin 7 - Actin cross-organization is required for axonal association of Schwann cells
}

\author{
Alejandro D. Roth ${ }^{1 *}$, Dalinda Liazoghli², Felipe Perez De Arce ${ }^{1}$, David R. Colman² (†) \\ (1) Laboratorio de Biología Celular de Glias, Departamento de Biología, Facultad de Ciencias, Universidad de Chile. Santiago, Chile \\ (2) Montreal Neurological Institute, McGill University. Montreal Canada \\ (†) Deceased
}

\begin{abstract}
Myelin sheaths present two distinct domains: compacted myelin spirals and flanking non-compacted cytoplasmic channels, where lipid and protein segregation is established by unknown mechanisms. Septins, a conserved family of membrane and cytoskeletal interacting GTPases, form intracellular diffusion barriers during cell division and neurite extension and are expressed in myelinating cells. Septins, particularly septin 7 (Sept7), the central constituent of septin polymers, are associated with the cytoplasmic channels of myelinating cells. Here we show that Schwann cells deprived of Sept7 fail to wrap around axons from dorsal root ganglion neurons and exhibit disorganization of the actin cytoskeleton. Likewise, Sept7 distribution is dependent on microfilament but not microtubule organization.
\end{abstract}

Key words: Axon wrapping, Septins, Myelination, Schwann Cells.

\section{INTRODUCTION}

Myelin sheathing of axons limits membrane depolarization to the unmyelinated areas regularly spaced along the axon, the nodes of Ranvier, resulting in a saltatory (jumping) conduction of nerve impulses. This characteristic reduces both the time and energy required for signals to transverse the axon while maintaining relatively small axonal diameter and maximizing neural tissue compaction (see reviews by Hartline and Colman 2007; Nave 2010). Myelination in mammals is carried out by two distinct cell lineages: neural crest-derived Schwann cells (SCs) in the peripheral nervous system (PNS), where one cell surrounds one axon (forming one internodal domain), and oligodendrocytes (OLGs) in the central nervous system (CNS), where each cell wraps multiple axons and forms multiple internodes. While myelin-specific proteins differ in these cell types, the general structure and organization of the sheath is similar, maintaining two specific domains: compact myelin, the major component of internodes, which is formed by a spiraled glial process from which most of the cytoplasm between the plasma membrane sheaths is extruded; and the areas that still contain cytoplasm, the non-compacted cytoplasmic channels, which endow cytoplasmic continuity to internodes, providing metabolic support to the myelin sheath (see reviews by Popko (2003) and Sherman and Brophy, (2005)).

Significant progress has been achieved in understanding the signaling mechanisms that allow migrating SCs to align and wrap axonal bundles, selecting from among them those that are to be individually sheathed and myelinated (see review by Court et al., (2006)). Here, SCs extend cell processes that advance through actin-dependent growth cone-like structures along two different planes: axial processes that align to the major axonal axi, and radial processes, formed in response to Rac1 activation, effecting the segregation of the axons to be myelinated from among other axons located in an axon bundle (Nodari et al, 2007).
The mechanisms that allow SC to establish different intracellular domains, be it between the areas that will lead to the extension of axial or radial processes or that distinguish compacted from non-compacted myelin have not yet been clarified, as shown in multiple knock-out animals that retain myelin sheath compaction in spite of the loss of the major proteins responsible for myelin compaction (Stoffel et al. 1997; Uschkureit et al. 2000) The presence of multiple septin isoforms in various proteomic characterizations of compact myelin (Dhaunchak et al. 2010; Roth et al. 2006; Taylor et al. 2004) has suggested a relevant role of these membrane and cytoskeletonassociated GTP-binding proteins that have been shown to participate in cell division and polarity by establishing intracellular cell diffusion barriers to proteins and mRNA through their interactions with membrane and cytoskeletal elements (see reviews by Barral and Mansuy 2007; Barral et al. 2000; Mostowy and Cossart 2012).

As members of the P-Loop superfamily of NTPases, septins present a conserved central core that includes the nucleotide-binding domain. The thirteen mammalian septin genes (plus their multiple splicing isoforms) can be grouped into three or four subgroups (see reviews cited above); and while the number and the precise stoichiometry of the different subunits that form septin polymers remain to be clarified, there is an emerging consensus as to the required presence of some isoforms (Kim et al., 2012). Septins have been shown to regulate microtubule stability (Kremer et al. 2005), while complexes containing Sept6/Sept7 mediate microtubule recruitment into axonal branches ( $\mathrm{Hu}$ et al., 2012). Likewise, other authors have shown that Sept $2 / 6 / 7$ complexes specifically associate and co-precipitate with the actin cytoskeleton (Kinoshita et al. 2002; Kinoshita et al. 1997).

Septin 7 (Sept7), a central constituent of most septin polymers (Sirajuddin et al. 2007), is associated with the cytoplasmic channels of myelinating SC, including the Schmidt-Lanterman incisures (Roth et al. 2006). Likewise, other 
septins (Sept2 and Sept6) have now been shown to localize to the non-compacted cytoplasmic channels of myelinating cells (both OLG and SC) while complexes made up of septins 2, 6 and 7 were shown to interact with MAL (Myelin and Leukocyte Associated protein), a proteolipid protein involved in the sorting of lipid-rich membrane micro-domains of myelinating cells (Buser et al. 2009).

In order to evaluate Sept7 function in myelinating cells, we established co-cultures between Dorsal Root Ganglia (DRG)-derived neurons and sciatic SCs transfected with Sept7-specific shRNAi. We show here that inhibition of Sept7 expression results in the disorganization of actin microfilaments, which likely mediates the alteration of Schwann cell shape and their ability to align and wrap around axons from dorsal root ganglia-derived neurons. Conversely, microfilament depolymerization by pharmacological agents alters Sept7 distribution from a gauze-like configuration to the formation of intracellular tubules. These results document the involvement of septin -7 actin interaction during the initial stages of SC association to axons.

\section{MATERIALS AND METHODS}

Unless noted, all chemicals were obtained from Sigma / Aldrich (St. Louis, MO) and all cell culture reagents were from Life Technologies (CA, USA).

Antibodies: Rabbit polyclonal serum against Sept7 was a kind gift of Sr. Shu-Chan Hsu (RUTGERS, State University of New Jersey). Western blotting was also asayed using a rabbit polyclonal serum against Sept7 obtained from Sigma/ Aldrich (St. Louis, MO). Rabbit and guinea pig polyclonal antiCaspr sera were generated against a GST-fusion protein of rat Caspr cytoplasmic domain as described previously (Tait et al. 2000). Rabbit polyclonal sera against DM20/PLP, E-cadherin, MBP and MAG have been characterized elsewhere (Fannon and Colman 1996; Pedraza et al. 1997). Mouse monoclonal antibodies against medial neurofilament were from Sigma/ Aldrich (St. Louis, MO), while mouse monoclonal antibodies against Rip were obtained from the Developmental Studies Hybridoma Bank at the University of Iowa (USA). Speciesspecific secondary antibodies conjugated to Alexa dyes, and Rodamine Red X-conjugated phalloidin were from Molecular Probes (Life Technologies. CA. USA). Primary cell cultures were initiated from E17 (for DRGs) or P1 (for SCs) SpragueDawley pups. All animal procedures were performed in accordance with CCAC guidelines and were approved by the bioethics committee of the Faculty of Science of the Universidad de Chile.

Plasmids: Palmitoyl-associated Enhanced Green Fluorescent Protein (Palmitoyl-EGF) expression plasmid was a kind gift from Dr. Weisong Shan (Montreal Neurological Institute, McGill University, Montreal, Canada). pSM2c plasmids from OpenBiosystems (USA) coded for a noninhibiting shRNA (control plasmid) or a septin7-specific inhibiting short hairpin RNA (shRNAi) corresponding to Entrez Probe ID: Pr182451.1 (OpenBiosystems Oligo id\# V2MM_73784) specific for both rat Sept7 mRNA transcription variants (positions 1321 through 1343 of variant 1 and 1324 through 1346 of variant 2).

Cell culture and transfection: Dorsal root ganglia neurons (DRGs) were cultured according to the protocol described by (Svenningsen et al. 2003). Briefly, E15/16 pregnant rats were sacrificed by $\mathrm{CO}_{2}$ inhalation. Embryos were removed, placed into cold L15 media and dissected under a flow hood. Embryo vertebral columns were transferred to clean L15, where spinal cords were retrieved along with adherent DRGs, which were plucked, trypsinized for 15 minutes at $37^{\circ} \mathrm{C}$ and dissociated by gentle aspiration through Pasteur pipettes. Trypsin was quenched by adding $10 \%$ volume of calf serum, cells were spun down at $800 \mathrm{rpm}$ and resuspended in Neurobasal medium supplemented with $2 \%$ B27 and 0.3\% L-glutamine (sNeurobasal) and counted. 50,000 cells $/ \mathrm{ml}$ were plated on coverslips pre-coated with $10 \mu \mathrm{g} / \mathrm{ml}$ poly-L-lysine or a $1 / 20$ dilution of Matrigel (Becton-Dickinson, USA). Glia-free DRG neuron cultures were generated by adding $5 \mu \mathrm{M}$ cytosine arabinoside (AraC) to the culture medium during the first 24 hours after plating. Myelinating cultures were generated from SC-containing DRG cultures that received $50 \mu \mathrm{g} / \mathrm{ml}$ of ascorbic acid after 7 days in vitro (DIV).

Sciatic nerve-derived SCs were obtained from P1 rats as described (Wood 1976). Briefly, animals were cold anesthetized on ice, followed by swift decapitation. Hindquarters were pinned and sciatic nerves were dissected and placed in cold L15 media. Nerves were stripped of their perineurium under a dissection microscope and transferred to L15 + $0.1 \%$ collagenase (Sigma, USA) and incubated at $37{ }^{\circ} \mathrm{C}$ for 30 minutes. Any remaining perineurium was dissected out and nerves were digested in L15 supplemented with $0.1 \%$ collagenase and $0.25 \%$ trypsin for 30 minutes. Calf serum was added (10\% of the final volume), nerves were dissociated with flamed Pasteur pipettes and the resulting cells spun down at 2,000 rpm for 1 minute. Cells were resuspended in sNeurobasal medium and counted. 1 million cells were transferred to a $1.5 \mathrm{ml}$ tube, incubated for 5 minutes with 2 $\mu \mathrm{g}$ of shRNAi plasmid against Sept7 or non-inhibiting shRNA control plasmid and $1 \mu \mathrm{g}$ of palmytoilated GFP expression plasmid. The cell suspension was eletroporated using an Amaxa Nucleofector (Lonza, Basel, Switzerland) according to manufacturer`s instructions, using the O-3 program. Following electroporation cells received $1 \mathrm{ml}$ of sNeurobasal, were spun down 5 minutes (2000 rpm), resuspended in sNeurobasal medium and plated on Matrigel-coated coverslips or added to DRG cultures. Cytoskeletal depolymerization drugs were diluted in pre-warmed sNeurobasal and added immediately to cells. Controls included the ethanol required to solubilize the stock solutions of latrunculin or vinblastin.

Immunocytochemistry: Cell cultures were washed twice with warm PBS, fixed for $10 \mathrm{~min}$ in $4 \%$ paraformaldehyde and washed 3 times in 1X PBS. Non-specific sites were blocked by incubating for at least 1 hour in blocking solution $(10 \%$ normal goat serum, $0.1 \% \mathrm{Tx}-100$ in $1 \mathrm{X}$ PBS). The indicated primary antibodies in blocking solution were incubated overnight at room temperature, washed briefly in 1X PBS, and species-specific secondary antibodies conjugated to Alexa488, Alexa543 or Alexa647 in blocking solution were incubated for 60 minutes at room temperature. The sections were washed 3 times in 1X PBS for 10 minutes, and mounted on with antifade medium (ProLong, Molecular Probes, Life Technologies, USA). Preparations were examined under 40 and 63X objectives with a scanning confocal microscope (Olympus Fluoview 1000 or Zeiss 510-Meta).

Immunoblotting: 48 hours post-transfection, isolated SC cultures (see above) were washed twice with $1 \mathrm{X}$ PBS $\left(37^{\circ} \mathrm{C}\right)$ and lysed with $100 \mu \mathrm{l}$ of RIPA lysis buffer (50mM Tris, $150 \mathrm{mM}$ 
$\mathrm{NaCl}, 0.1 \%$ SDS, $0.5 \%$ sodium deoxycholate, $1 \%$ Triton $\mathrm{X} 100$; 1X EDTA-Free protease inhibitor cocktail, (Roche, Germany)). Protein concentrations were determined by micro BCA (Pierce Chemical Co) and $5 \mu \mathrm{g}$ proteins were subjected to PAGE in $12 \%$ SDS Gels. Proteins were transferred to a nitrocellulose membrane and blocked with 5\% milk and $0.2 \%$ Tween 20 in 1X TBS (blocking solution) for 2-3 hours. Membranes were incubated overnight with the indicated primary antibodies in blocking solution, washed and incubated with speciesspecific secondary antibodies conjugated to HRP (Roche, Germany) in blocking solution. After extensive washes, proteins were detected by chemiluminescence reaction (Amersham) and signal optical density assessed using ImageJ software (Schneider et al., 2012). Relative protein levels were normalized to the proteins transferred to the blotting membrane stained by Ponceau S staining (see Romero-Calvo et al., 2010 for full protocol). It should noted that no significant variations in normalization were observed when the Ponceau $\mathrm{S}$ densitometry used was that of a single band (100 KDa or 180 $\mathrm{KDa}$ ) or the densitometric value obtained for the whole lane.

\section{RESULTS}

Septin 7 inhibition does not prevent Schwann cell attachment to substrate.

Sept7 is profusely expressed in embryonic and post-natal neural tissues, particularly in the sciatic nerve (Figure 1A), suggesting that its inhibition may decrease Schwann cell viability. Nevertheless, when sciatic nerve-derived SCs were co-transfected with plasmids encoding septin7-inhibiting shRNAi and palmitoyl-EGFP and seeded on ECM-gel coated plates, we observed that Sept7 inhibition did not reduce cell viability or substrate attachment (Figure 1B). Compared to controls, shRNAi transfected SC cultures presented $40 \%$ reduction in overall septin7 levels (Figure 1C, quantified in D), matching the transfection efficiency observed as the expression of EGFP of SCs in culture ( $40 \%$ of cells express EGFP). As expected, SCs co-transfected with EGFP expression plasmid and non-inhibiting shRNAi (control plasmid) did not present alterations in Sept7 expression or any evident morphological effects.

Sept7 inhibition abolishes Schwann cell association to axons.

DRG cultures are a well-recognized model of in vitro myelination (Jarjour et al. 2012; Svenningsen et al. 2003); where endogenous SCs can be eliminated by a 24 hour incubation in the presence of $5 \mu \mathrm{M}$ AraC. After a thorough wash with cell culture medium, the axons of the DRG neurons are disposed for unrestricted access from exogenous SCs. We observed that when exogenous SCs were transfected with control plasmids (non-inhibiting shRNA and palmitoyl-EGFP) these cells aligned, extended and spiraled over axons (Figure 2A control), which constitute the initial steps, recognition and adhesion required for myelin sheath formation. Contrarily, when Sept7 expression of exogenous SC was inhibited through transfection with shRNAi, cells acquired a flattened morphology and failed to align to or wrap around axons (Figure 2A, shRNAi).

Endogenous SCs can be induced to myelinate resident axons in DRG cultures by supplementing the cell culture medium with ascorbic acid $(20 \mu \mathrm{M})$ for 10 days. When such cultures receive freshly isolated SCs, the new arrivals colonize axonal segments and displace resident cells, extending long processes and attempting to wrap around the axons in the culture (Figure 2B). These freshly added cells compete with the resident SCs over axon segments devoid of myelin (Sims and Gilmore 1989), while myelinated segments (shown by MAG staining) are not subjected to novel wrapping. Exogenous SCs in which Sept7 expression was inhibited by shRNAi did not align to axons, acquired a flattened form and were unable to displace resident SC from axons (Figure 2 shRNAi).

Considering the reported interaction between septin oligomers and both the actin and tubulin cytoskeletons (Barral et al. 2000; Kinoshita et al. 2002; Kinoshita et al. 1997; Kremer et al. 2005; Sirajuddin et al. 2007), we assayed the consequences of the inhibition of Sept7 expression on the organization of SC cytoskeletal components. We observed that Sept7 inhibition promoted the loss of the distinct microfilaments observed in control SCs (Figure 2C). Actin microfilaments of control SCs (non-inhibiting shRNAi) and of non-transfected SCs remaining in the Sept7-inhibited SCs (both cell types marked with double arrowheads) form a web that runs parallel to the cell's major axis and organizes into well defined structures around the cell nucleus (Figure 2C). In contrast, transfected SCs that present an evident loss of Sept7 expression display diffuse actin staining, lack of clearly defined actin filaments and a disarray of their actin cytoskeleton (Figure 2C).

The observed impact of Sept7 inhibition on SC actin cytoskeleton prompted us to characterize Sept7 distribution in response to cytoskeleton modifications. As observed in Figure 3 , actin filament depolymerization induced by latrunculin causes a significant reorganization of Sept7. While Sept7 in control SCs are organized mostly as filamentous structures ("gauze-like", figure 3A control) which distribute in most of the cell body and run parallel to the actin microfilaments, latrunculin-treated cells preferentially form circular or semicircular Sept7-rich rings with an approximate diameter of $1 \mu \mathrm{m}$ (figure $3 \mathrm{~A}$ latrunculin). The notorious change in Sept7 organization is a specific consequence of the loss of microfilament structure, and was not observed upon microtubule depolymerization induced by vinblastin (100 $\mathrm{nM}, 30 \mathrm{~min}$. Figure 3C, compare control and vinblastin). Nevertheless, the alteration of microtubule dynamics in response to vinblastin can still result in alterations in the distribution of Sept7, as appears to be the case in Figure 3C. Still, these effects are less evident than the changes that ensue from microfilament depolymerization.

Sept7-actin interdependence was maintained in spite of myelin sheath establishment. Myelinated DRG-SC co-cultures incubated with $20 \mu \mathrm{M}$ latrunculin presented diffuse actin distribution throughout the cell (figure 3B), accompanied by Sept7 redistribution into punctate structures that did not line the non-compacted cytoplasmic channels observed in control cells.

\section{DISCUSSION}

Septins localize to non-compacted cytoplasmic channels of myelinating cells in the CNS and PNS, including the Schmidt-Lanterman incisures and the Cajal bands of SC (Buser et al. 2009; Roth et al. 2006). Sept7 plays a central role in the formation of septin macro-complexes, as highlighted by its effect on lymphocyte motility, where the inhibition of 
this isoform results in the loss of the entire septin complex, resulting in an alteration of cell morphology and motility (Tooley et al. 2009). We show here that Sept7 is required for axonal attachment by SC, probably through its codependent interaction with the actin cytoskeleton.

When we assayed SC morphology in the absence of axons we did not observe any obvious morphological changes in Sept7-inhibited SCs, a difference that might be ascribed to the failure to obtain stable transfectants, which has been attributed to the importance of Sept7 in SC survival (Buser et al. 2009), precluding a long-term study of Sept7-inhibited SC. However, we cannot rule out that Sept7 protein expression was not completely inhibited and that cells capable of interacting with axons presented residual Sept7 levels, below the detection limit of immunofluorescence. As observed in Figure 2, Sept7inhibited cells acquired multiple shapes, which might indicate different levels of inhibition. Likewise, we did not evaluate the levels of expressions of other septins, which could be proposed to take up the function of Sept7, for it has been shown that the inhibition of Sept7 induces the loss of the whole septin complex (Toole et al., 2009).

Transient transfection impedes the observation of the direct effect of Sept7 on myelination; however, SC-axon interaction on a short time scale could still be assessed. Exogenous SC compete over unmyelinated axonal segments in vivo (Sims and Gilmore 1989), thus when added to myelinated DRG cultures
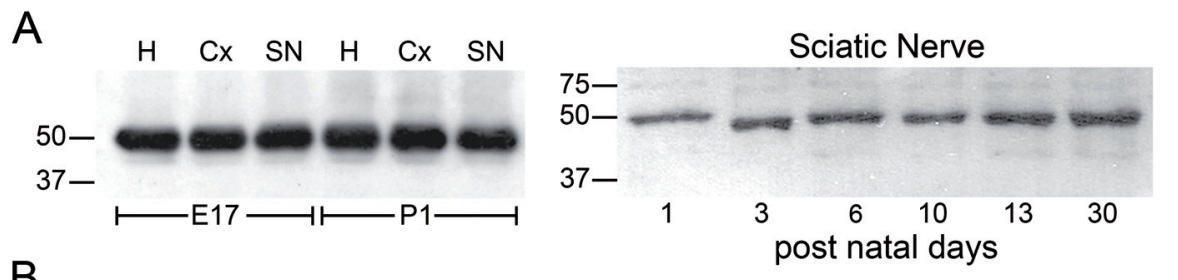

B
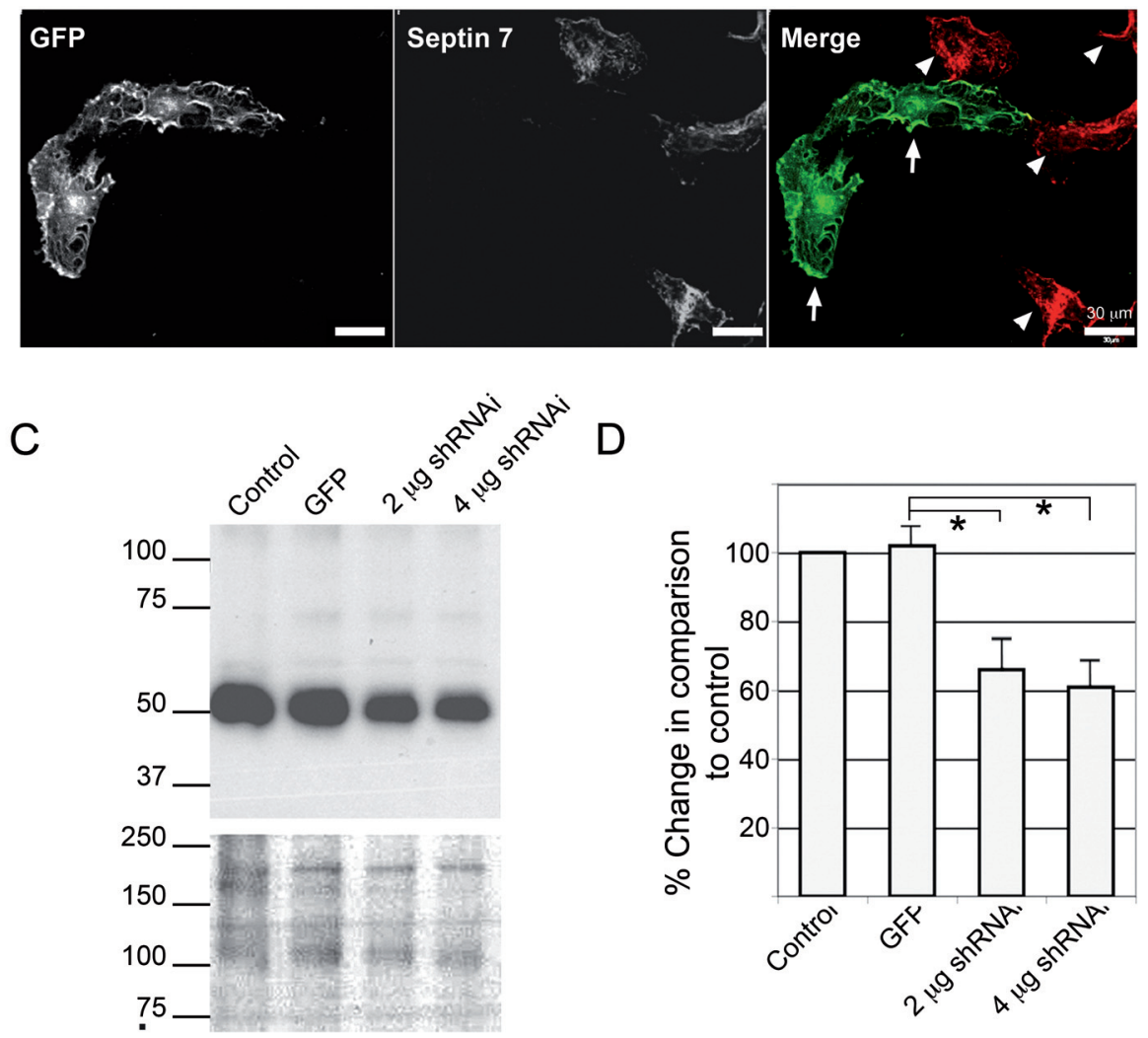

Figure 1: Septin 7, a sciatic nerve protein can be significantly inhibited in Schwann cells transfected with sequence-specific ShRNAi.

A: Sept7 is abundant in perinatal and postnatal rat sciatic nerves and nervous tissues (H: hippocampus, Cx: Cortex, SN:Sciatic nerve. Transient transfection of 1.5 million SC with Sept7-specific shRNAi reduces the overall presence of this protein after 48 hours of in vitro culture, as determined by immunofluorescence (B) and immunoblot (C) staining of Sept7. SC co-transfected with plasmids that encode a palmitoyl-associated GFP (green) and a shRNAi directed against Sept7 (arrows) do not present Sept7 staining, while cells that do not present GFP signal maintain Sept7 expression (arrowheads and red-labeled cells). Efficiency of Sept7 inhibition was not increased by a higher concentration of shRNAi during transfection, as shown by immunoblotting (C), where non-transfected cells (Control), cells transfected with non-inhibiting control shRNAi (GFP), and cells transfected with two concentrations of shRNAi ( 2 and $4 \mu \mathrm{g})$ cultured for 48 hrs prior to protein extraction. D: Quantification of Sept7 normalized by the total proteins transferred to the membrane and expressed as percent of expression relative to control ([OD sample]*100/[OD untransfected-control]) $\left({ }^{*}, P<0.05\right)$. 
control SC spiraled around axons and integrated into the cell culture, while cells transfected with Sept7-shRNAi attached to multiple axons but failed to elongate or spiral around them. Considering the various septin-cytoskeleton interactions described in the literature (Barral et al. 2000; Kinoshita et al. 2002; Kinoshita et al. 1997; Kremer et al. 2005; Sirajuddin et al. 2007), SC abnormal behavior and shape suggest an alteration of cytoskeletal functions.

We observed that Sept7 inhibition disrupted SC microfilaments, consistent with the loss of axonal wrapping in response to actin depolymerization elicited by cytochalasin
D treatments (Fernandez-Valle et al. 1997) and with the close interaction of septin 2 with actin and with integral myelin proteins (MAL) through Sept2/6/7 complexes (Buser et al. 2009). Conversely, actin filament depolymerization with latrunculin elicited a reorganization of Sept7 filaments into large $(0.6$ to $1.0 \mu \mathrm{m})$ circular or tubular structures previously observed for other septin family members (Kinoshita et al. 1997; Rodal et al. 2005; Schmidt and Nichols 2004). These septin rings have been proposed to arise from the re-association of septin filaments, which freed from their actin association, reflect the $25^{\circ}$ to $30^{\circ}$ angle observed in the

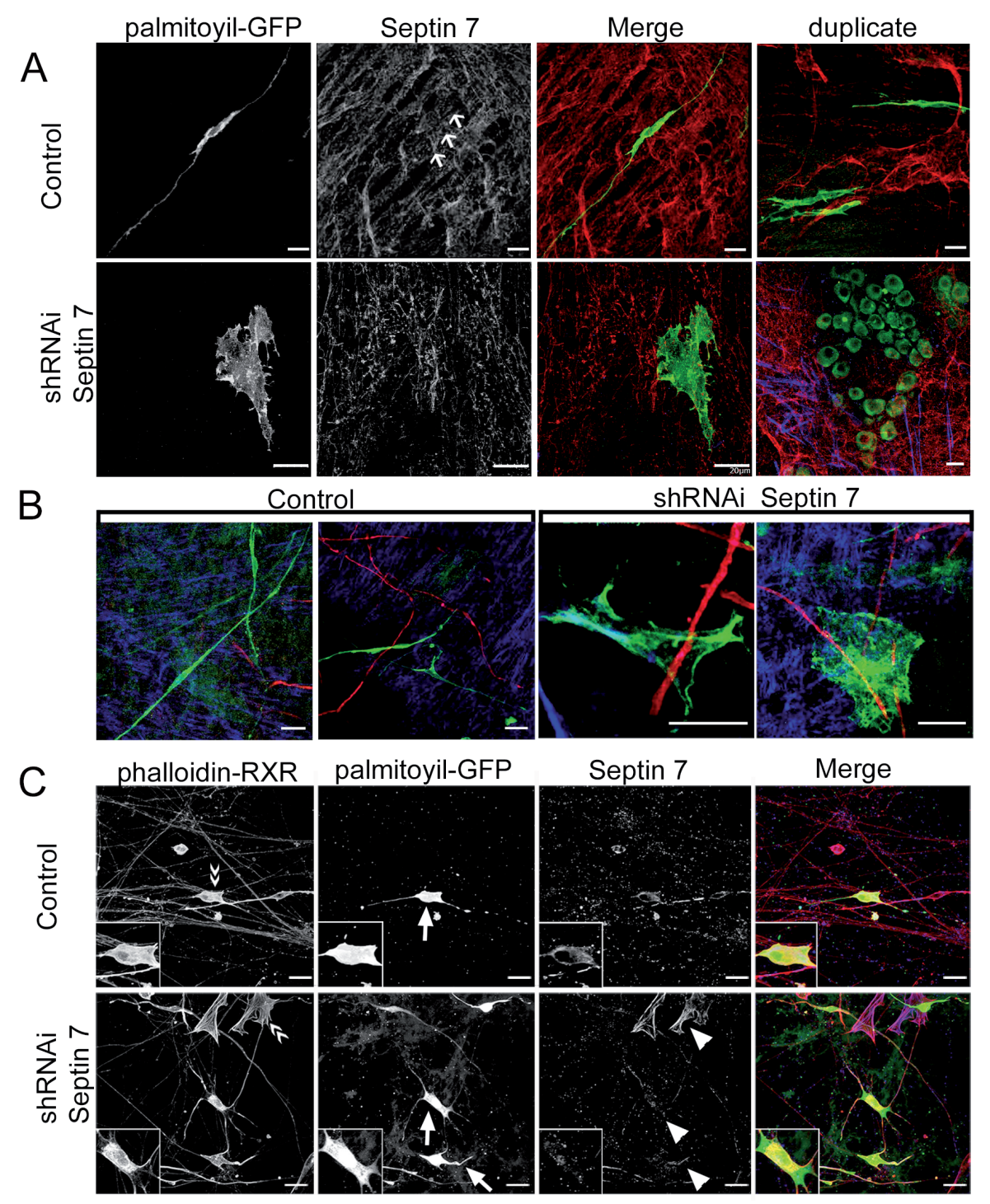

Figure 2: Sept7 inhibition blocks elongation of Schwann and prevents their association to axons in myelinated cell cultures by disorganizing the actin cytoskeleton.

(A) Isolated sciatic nerve derived SCs co-transfected with plasmids coding for palmitoyol-associated GFP and non-inhibiting control shRNA (Control) or Sept7-inhibiting shRNAi (shRNAi Sept7). Sept7 expression (open arrows) 48 hrs post-transfection assayed by immunofluorescence. (B) Freshly extracted sciatic nerve SCs transfected with plasmids coding for palmitoyil-bound GFP (green) and non-inhibiting control shRNA (Control) or Sept7-inhibiting shRNAi (shRNAi Sept7) were added to myelinating DRG-cultures where they competed for attachment to axons with resident SCs. Co-cultures were fixed and immunostained after 48 hrs. for Sept7 (blue) and MAC (Red). (C) Isolated sciatic nerve-derived SCs transfected with plasmids encoding control or Sept7-inhibiting shRNAi. The actin cytoskeleton was shown by Phalloidin-RRX (double arrowheads, red in merged images), expression of co-transfected plasmid coding for palmitoylated GFP (arrows, green in merged images). Sept7 expression as determined by immunofluorescence (arrowheads, blue in merged images). Scale bars: $20 \mu \mathrm{m}$. 
electron microscope images of Sept $2 / 6 / 7$ complexes / filaments (Gladfelter and Montagna 2007; Sirajuddin et al. 2007). It will be interesting to determine if the septin ring complexes from SC posses the same sub-units.

In the present study we have shown the cross-dependence between Sept7-containing structures and actin microfilaments during the initial stages of SC association to axons, the first stage in the organization of the axo-glial interaction that results in axonal myelination in the PNS.

\section{ACKNOWLEDGEMENTS}

This manuscript is dedicated to the memory of Dr. David R. Colman, who lead by example in the enjoyment of intellectual
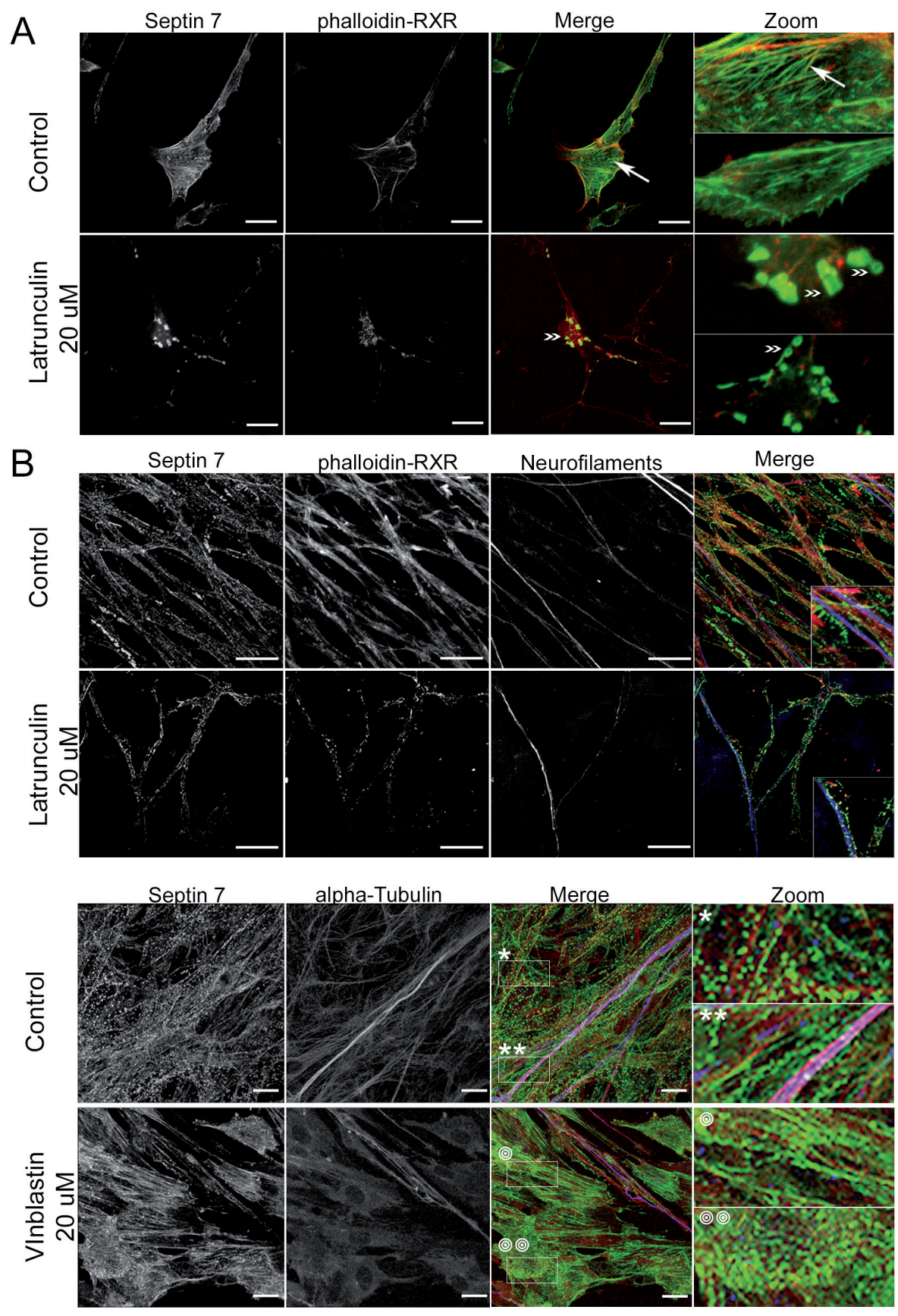

Figure 3: Actin depolymerization induces Sept7 reorganization from fibers into tubules.

(A) Schwann cell Sept7 organized in gauze-like configuration (arrows) associated to actin fibers (phalloidin-RXR) as fibers. Treatment with $20 \mu \mathrm{M}$ latrunculin for 120 min disrupts actin cytoskeleton and alters Sept7 organization, which form cytoplasmic tubules (double arrowheads). Merged images: Sept7- green, phalloidin-RRX in red. (B) Association to axons does not prevent Schwann cell-Sept7 reconfiguration in response to actin cytoskeleton disruption by latrunculin ( $20 \mu \mathrm{M}$ for $120 \mathrm{~min}$. (C) Disruption of microtubules by $20 \mu \mathrm{M}$ vinblastin for 120 minutes inhibits Sept7 gauze-like organization. Merged images: Sept7 colored green, Tubulin in red. Bars: $20 \mu m$. Zoom from merge images shows the coexistence of Sept7 fibers and puncta under control and vinblastin. 
pursuit. The authors would like to thank Dr. Juan Bacigalupo for his critical reading of the manuscript, Miss Gabriela Zavala for her technical assistance and Dr. Gabriela Mercado for her assistance with image processing. Funding for this research was provided by Myelin Repair Foundation Project (awarded to DRC, project \#MRF0104-DRC004) Fondecyt project \#1080252 (Conicyt, Chile) and ENL11/08 (Vicerrectoría de Investigación, Universidad de Chile), awarded to AR.

\section{REFERENCES}

BARRAL Y, MANSUY IM. (2007). Septins: cellular and functional barriers of neuronal activity. Curr Biol 17(22):R961-963.

BARRAL Y, MERMALL V, MOOSEKER MS, SNYDER M. (2000). Compartmentalization of the cell cortex by septins is required for maintenance of cell polarity in yeast. Mol Cell 5(5):841-851.

BUSER AM, ERNE B, WERNER HB, NAVE KA, SCHAEREN-WIEMERS N. (2009). The septin cytoskeleton in myelinating glia. Mol Cell Neurosci 40(2):156-166.

COURT FA, WRABETZ L, FELTRI ML. (2006) Basal lamina: Schwann cells wrap to the rhythm of space-time. Curr Opin Neurobiol. 16(5):501-7.

DHAUNCHAK AS, HUANG JK, DE FARIA JR O, ROTH AD, PEDRAZA L, ANTEL JP, BAR-OR A, COLMAN DR. (2010). A proteome map of axoglial specializations isolated and purified from human central nervous system. Glia 58(16):1949-1960.

FANNON AM, COLMAN DR. (1996). A model for central synaptic junctional complex formation based on the differential adhesive specificities of the cadherins. Neuron 17(3):423-434.

FERNANDEZ-VALLE C, GORMAN D, GOMEZ AM, BUNGE MB. (1997). Actin plays a role in both changes in cell shape and gene-expression associated with Schwann cell myelination. J Neurosci 17(1):241-250.

GLADFLETER AS, MONTAGNA C. (2007). Seeking truth on Monte Verita. Workshop on the molecular biology and biochemistry of septins and septin function. EMBO Rep 8(12):1120-1126.

HARTLINE DK, COLMAN DR. (2007). Rapid conduction and the evolution of giant axons and myelinated fibers. Curr Biol 17(1):R29-35.

HU J, BAI X, BOWEN JR, DOLAT L, KOROBOVA F, YU W, BAAS PW, SVITKINA T, GALLO G, SPILIOTIS ET. (2012) Septin-driven coordination of actin and microtubule remodeling regulates the collateral branching of axons. Curr Biol. 22(12):1109-15.

JARJOUR AA, ZHANG H, BAUER N, FFRENCH-CONSTANT C, WILLIAMS A. (2012). In vitro modeling of central nervous system myelination and remyelination. Glia 60(1):1-12.

KINOSHITA M, FIELD CM, COUGHLIN ML, STRAIGHT AF, MITCHINSON TJ. (2002). Self- and actin-templated assembly of Mammalian septins. Dev Cell 3(6):791-802.

KINOSHITA M, KUMAR S, MIZOGUCHI A, IDE C, KINOSHITA A, HARAGUCHI T, HIRAOKA Y, NODA M. (1997). Nedd5, a mammalian septin, is a novel cytoskeletal component interacting with actin-based structures. Genes Dev 11(12):1535-1547.

KREMER BE, HAYSTEAD T, MACARA IG. (2005). Mammalian septins regulate microtubule stability through interaction with the microtubulebinding protein MAP4. Mol Biol Cell 16(10):4648-4659.

KIM MS, FROESE CD, XIE H, TRIMBLE WS (2012) Uncovering principles that control septin-septin interactions. J. Biol Chem. 287(36):30406-13.

MOSTOWY S, COSSART P. (2012). Septins: the fourth component of the cytoskeleton. Nat Rev Mol Cell Biol 13(3):183-194.
NAVE KA. (2010). Myelination and the trophic support of long axons. Nat Rev Neurosci 11(4):275-283.

NODARI A, ZAMBRONI D, QUATTRINI A, COURT FA, D'URSO A, RECCHIA A, TYBULEWICZ VL, WRABETZ L, FELTRI ML. (2007) Beta1 integrin activates Rac1 in Schwann cells to generate radial lamellae during axonal sorting and myelination. J Cell Biol. 177(6):1063-75.

PEDRAZA L. FIDLER L, STAUGAITIS SM, COLMAN DR. (1997). The active transport of myelin basic protein into the nucleus suggests a regulatory role in myelination. Neuron 18(4):579-589.

POPKO B. (2003). Myelin: not just a conduit for conduction. Nat Genet 33(3):327-328.

RODAL AA, KOZUBOWSKI L, GOODE BL, DRUBIN DG, HARTWIG JH. (2005). Actin and septin ultrastructures at the budding yeast cell cortex. Mol Biol Cell 16(1):372-384.

ROMERO-CALVO I, OCÓN B, MARTÍNEZ-MOYA P, SUÁREZ MD ZARZUELO A, MARTÍNEZ-AGUSTIN O, SANCHEZ DE MEDINA F. (2010). Reversible Ponceau staining as a loading control alternative to actin in Western blots. Analytical Biochemistry 401(2):318-320.

ROTH AD, IVANOVA A, COLMAN DR. (2006). New observations on the compact myelin proteome. Neuron Glia Biol 2(1):15-21.

SCHNEIDER CA. RASBAND WS. ELICEIRI KW. (2012) NIH Image to ImageJ: 25 years of image analysis. Nature Methods 9: 671-675

SCHMIDT K, NICHOLS BJ. (2004). Functional interdependence between septin and actin cytoskeleton. BMC Cell Biol 5(1):43.

SHERMAN DL, BROPHY PJ. (2005). Mechanisms of axon ensheathment and myelin growth. Nat Rev Neurosci 6(9):683-690.

SIMS TJ, GILMORE SA. (1989). Interactions between Schwann cells and CNS axons following a delay in the normal formation of central myelin. Exp Brain Res 75(3):513-522.

SIRAJUDDIN M, FARKASOVSKY M, HAUER F, KUHLMANN D MACARA IG, WEYAND M, STARK H, WITTINGHOFER A. (2007). Structural insight into filament formation by mammalian septins. Nature 449(7160):311-315

STOFFEL W, BOISON D, BUSSOW H. (1997). Functional analysis in vivo of the double mutant mouse deficient in both proteolipid protein (PLP) and myelin basic protein $(\mathrm{MBP})$ in the central nervous system. Cell Tissue Res 289(2):195-206.

SVENNINGSEN AF, SHAN WS, COLMAN DR, PEDRAZA L. 2003. Rapid method for culturing embryonic neuron-glial cell cocultures. J Neurosci Res 72(5):565-573.

TAIT S, GUNN-MOORE F, COLLINSON JM, HUANG J, LUBETZKI C, PEDRAZA L, SHERMAN DL, COLMAN DR, BROPHY PJ. (2000). An oligodendrocyte cell adhesion molecule at the site of assembly of the paranodal axo-glial junction. J Cell Biol 150(3):657-666.

TAYLOR CM, MARTA CB, CLAYCOMB RJ, HAN DK, RASBAND MN, COETZEE T, PFEIFFER SE. (2004). Proteomic mapping provides powerful insights into functional myelin biology. Proc Natl Acad Sci U S A 101(13):4643-4648.

TOOLEY AJ, GILDEN J, JACOBELLI J, BEEMILLER P, TRIMBLE WS, KINOSHITA M, KRUMMEL MF. (2009). Amoeboid T lymphocytes require the septin cytoskeleton for cortical integrity and persistent motility. Nat Cell Biol 11(1):17-26

USCHKUREIT T, SPORKEL O, STRACKE J, BUSSOW H, STOFFEL W. (2000). Early onset of axonal degeneration in double (plp-/-mag-/-) and hypomyelinosis in triple (plp-/-mbp-/-mag-/-) mutant mice. J Neurosci 20(14):5225-5233.

WOOD PM. (1976). Separation of functional Schwann cells and neurons from normal peripheral nerve tissue. Brain Res 115(3):361-375. 
